Z Gerontol Geriat 2018 $51: 741-743$ https://doi.org/10.1007/s00391-018-01462-7 Online publiziert: 17. Oktober 2018

(c) Springer Medizin Verlag GmbH, ein Teil von Springer Nature 2018

CrossMark

\author{
Walter Hewer $\cdot$ Vjera Holthoff-Detto ${ }^{2,3}$. Daniel Kopf ${ }^{4}$ \\ ${ }^{1}$ Klinik für Gerontopsychiatrie, Klinikum Christophsbad, Göppingen, Deutschland \\ ${ }^{2}$ Klinik für Psychiatrie, Psychotherapie und Psychosomatik, Alexianer Krankenhaus Hedwigshöhe, Berlin, \\ Deutschland \\ ${ }^{3}$ Klinik und Poliklinik für Psychiatrie und Psychotherapie, Medizinische Fakultät, Technische Universität \\ Dresden, Dresden, Deutschland \\ ${ }^{4}$ Geriatrische Klinik, Kath. Marienkrankenhaus GmbH, Hamburg, Deutschland
}

\title{
Themenschwerpunkt alt gewordene psychisch Kranke
}

natsprävalenz psychischer Störungen von 35,2\% ermittelt wurde [2], zeigt jedoch, dass das Merkmal „schwere psychische Erkrankung“ eine relativ kleine Gruppe von Patienten umschreibt, der aber aufgrund der Schwere der Beeinträchtigung und des daraus resultierenden medizinischen und psychosozialen Versorgungsbedarfs eine hohe Bedeutung zukommt.

Auch wenn seit Langem bekannt ist, dass das Vorliegen einer schweren psychischen Erkrankung mit einer beträchtlichen Verkürzung der Lebenserwartung von einer Dekade (und mehr) verbunden ist [4], ist aus demografischen Gründen von einer steigenden Zahl Betroffener in unserer Gesellschaft auszugehen. Nachdem in den zurückliegenden Jahren einige Themenschwerpunkte bereits psychische Alterserkrankungen zum Gegenstand hatten (Demenz, Depression, posttraumatische Störungen), scheint es an der Zeit, sich nun den hier angesprochenen Krankheitsbildern zuzuwenden. Dies geschieht aus einer primär klinischen Perspektive; sämtliche Autoren der Beiträge, in die nachfolgend kurz eingeführt wird, verfügen über langjährige Erfahrungen in den jeweiligen Bereichen.

Erkrankungen des schizophrenen Formenkreises (Autoren C. Jagsch und A. Hofer) sind, bei enger Falldefinition, mit einer Prävalenz von etwas unter $1 \%$ zwar relativ seltene, jedoch aufgrund der im Durchschnitt hohen Krankheitslast und der auf Behandlerseite geforderten spezifischen diagnostischen und therapeutischen Kompetenzen wichti- ge Erkrankungen. Die Autoren rufen Kernmerkmale dieser überwiegend in der ersten Lebenshälfte beginnenden Störungsbilder in Erinnerung und benennen mit frühem, späterem oder sehr spätem Krankheitsbeginn korrelierende Merkmale. Diese sind jedoch in der konkreten Behandlungssituation häufig so nicht nachvollziehbar, angesichts einer hohen Variabilität der Krankheitsverläufe, abhängig von individuellen bzw. krankheitsbezogenen Faktoren, und nicht zuletzt auch komplizierenden demenziellen Prozessen. Dabei sollten neben Verläufen, die bis ins hohe Lebensalter mit einer sehr schweren Symptomatik einhergehen, relativ günstige Entwicklungen bis hin zu Remissionen nicht unbeachtet bleiben.

J. Benninghoff und P. Brieger geben einen Überblick zu den bipolaren Störungen. Diese manifestieren sich ebenfalls deutlich häufiger im jüngeren Erwachsenenalter, gleichwohl muss die Möglichkeit eines Auftretens in der zweiten Lebenshälfte bedacht und es müssen dann körperliche und pharmakogene Ursachen besonders beachtet werden. Neben der klassischen Bipolar-I-Störung sind, gerade bei Älteren, auch Bipolar-IIStörungen relevant. Diese können leicht übersehen werden, da im langjährigen Krankheitsverlauf häufig depressive Phasen dominieren und andererseits hypomanische - also nicht dem Vollbild einer Manie entsprechende - Zustände nicht immer als solche erkannt werden. Wegen der wesentlich von der unipolaren Depression abweichenden alle Schweregrade hinweg eine Zwölfmo- 
Behandlung sollte dieses Krankheitsbild jedoch Geriatern bekannt sein, auch im Hinblick darauf, dass der bipolare Charakter der affektiven Störung nicht selten erst im vorgerückten Lebensalter erkennbar wird.

Gegenstand des Beitrags von D. Wolter sind die Suchterkrankungen, wobei neben den „Klassikern“ Alkohol- und Medikamentenabhängigkeit auch Störungen infolge illegaler Drogen und die sog. Verhaltenssüchte an Bedeutung gewinnen. Wegen der evidenten Konsequenzen für die körperliche Gesundheit wird auch die Tabakabhängigkeit eingehend thematisiert. Der Verfasser arbeitet die besonderen Risikomerkmale heraus, die bei Suchterkrankungen mit „early onset" zu beachten sind. So sehr eine Abstinenz anzustreben ist, darf wie der Autor hervorhebt - das Nichterreichen dieses Ziels nicht zu einem therapeutischen Nihilismus führen, da im Sinne einer "harm reduction“" auch unterhalb dieser hohen Messlatte für den Gesundheitszustand der Patienten relevante Ergebnisse erreicht werden können. Für die Altersmedizin von besonderem Interesse sind schließlich auch die komplexen Zusammenhänge zwischen Suchterkrankungen und neurokognitiven Störungen, insbesondere Demenzen, die in dem Beitrag umrissen werden.

C. Lange-Asschenfeldt hebt hervor, dass Psychopharmakotherapie bei alt gewordenen Menschen mit schweren psychischen Erkrankungen die Einbindung in ein multimodales, biopsychosozial ausgerichtetes Behandlungskonzept voraussetzt. Bei der Indikationsstellung sind die aus der Gerontopharmakologie gut bekannten Gefährdungen in einer Nutzen-Risiko-Bewertung gegenüber der Schwere der Beeinträchtigungen, die durch die medikamentöse Therapie beeinflusst werden können, abzuwägen. Auf dieser Basis werden konkrete Empfehlungen für die altersgerechte Anwendung der wichtigsten Psychopharmaka ausgesprochen. Dabei gilt, dass bei besprochenen psychischen Erkrankungen der Stellenwert der Psychopharmakotherapie deutlich weiter reicht als bei den kognitiven Störungen (also Demenz und Delir) der Fall. Während bei den letzt- genannten Störungsbildern Psychopharmaka fakultativ zum Einsatz kommen, gehören sie z.B. bei schizophrenen und schweren affektiven Erkrankungen zu den im Regelfall indizierten Verfahren, verbunden mit der Notwendigkeit einer fachärztlichen (Mit)Behandlung.

D. Kopf und W. Hewer fassen aktuelle Erkenntnisse zur körperlichen Gesundheit der Zielgruppe dieses Hefts zusammen und gehen auf einige in der altersmedizinischen Praxis besonders wichtige Komorbiditäten näher ein. Obwohl die Betroffenen im Vergleich zur Allgemeinbevölkerung signifikant höheren somatischen Risiken unterliegen, werden diese häufig nicht (genügend) wahrgenommen, v.a. dann, wenn die Patienten mit dem Merkmal (Stigma) „psychisch krank" vorgestellt werden bzw. gravierende psychische Beeinträchtigungen unübersehbar sind. Dieses jedem erfahrenen Psychiater gut bekannte und in der neueren angloamerikanischen Literatur als "diagnostic overshadowing“ bezeichnete Phänomen [12], sollte Anlass sein, unspezifischen Symptomen mit angemessener Aufmerksamkeit zu begegnen und vorschnelle Zuordnungen im Sinne einer "psychischen Ursache" zu vermeiden. Schließlich umreißen die Autoren Erklärungsansätze für die besprochenen Risikokonstellationen und skizzieren Wege, wie mit der damit verbundenen interdisziplinären Herausforderung umgegangen werden kann.

In allen Beiträgen kommt mehr oder weniger deutlich der Aspekt zum Ausdruck, dass spezifische empirische Evidenzen (also beispielsweise RCT), bezogen auf die Zielgruppe dieses Themenschwerpunkts, dünn gesät sind. Deshalb weisen die Autoren verschiedentlich darauf hin, dass diagnostisch und therapeutisch altersübergreifende Prinzipien gelten, mit den erforderlichen Anpassungen an die Besonderheiten der behandelten Gruppe von Patienten. Dies ist grundsätzlich so auch nachvollziehbar, insofern hier Störungsbilder mit Beginn in früheren Lebensphasen im Mittelpunkt stehen.

Andererseits kommt es im Alter in Verbindung mit den assoziierten somatischen und psychosozialen Prozessen re- gelhaft zu bedeutsamen oder auch richtungweisenden Veränderungen in den Krankheitsbildern, wie beispielsweise bei Überlagerungen durch demenzielle Prozesse der Fall. Deshalb ist angesichts einer wachsenden Zahl Betroffener zu hoffen, dass in den kommenden Jahren die Datenbasis durch Studien, die alt gewordene Menschen mit psychischen Erkrankungen in den Fokus rücken, verbreitert wird. Wünschenswert wären in dieser Hinsicht nicht zuletzt auch Verlaufsstudien, die einerseits den Übergang vom mittleren ins höhere Lebensalter und andererseits auch die Entwicklungen bis in die höchsten Altersgruppen verfolgen würden.

Damit würde ein wesentlicher Beitrag geleistet zu der Verbreiterung der jenseits demenzieller und depressiver Erkrankungen eher schmalen Datenlage zur Epidemiologie psychischer Störungen im (hohen) Alter [14]. Nicht zuletzt könnten solche Studien auch Erkenntnisse zur Prognose der hier besprochenen Erkrankungen liefern. Ohne deren Schwere mit den vielfältigen, weiter oben bereits angerissenen negativen Konsequenzen in Abrede zu stellen, sollte nicht aus dem Blickfeld geraten, dass im Langzeitverlauf eine Mitigierung der Symptomatik bis hin zu Remissionen möglich ist [10].

In dem Zusammenhang ist nicht zuletzt das Konzept der „recovery“ von Interesse, das in den letzten Jahren an Bedeutung gewonnen hat und einen Zustand beschreibt, in dem eine betroffene Person trotzkrankheitsbedingter Einschränkungen wieder eine grundsätzlich positive Lebensperspektive entwickeln konnte $[1,6]$. Dabei ist der Gedanke, dass Menschen, die bei Erkrankungen mit einer signifikant verkürzten Lebenserwartung ein höheres oder hohes Lebensalter erreicht haben, als „Überlebende“ betrachtet werden können, vielleicht auch von Interesse ([11]; s. auch Beitrag Wolter in diesem Heft).

Versorgungspolitisch ergeben sich in Bezug auf die Zielgruppe dieses Themenschwerpunkts beachtliche Herausforderungen:

- Menschen mit schweren psychischen Erkrankungen sind auf vielfältige professionell erbrachte Maßnahmen angewiesen, die neben einer allge- 
meinmedizinisch-geriatrischen und psychiatrischen Versorgung pflegerische Leistungen und Angebote seitens verschiedenster im psychosozialen Bereich tätiger Personen und Institutionen beinhalten (gesetzliche Betreuer, Beratungsstellen u.a.m.). Dies stellt eine erhebliche Herausforderung dar angesichts der Fragmentierung unseres Gesundheits- und Sozialsystems [3], aber auch im Hinblick darauf, dass die Betroffenen krankheitsbedingt häufig den Sinn der von professioneller Seite vorgeschlagenen Maßnahmen nicht oder nur eingeschränkt nachvollziehen.

- In Ergänzung zu den klassischen psychiatrischen therapeutischen und rehabilitativen Angeboten zeichnet sich in den letzten Jahren ab, dass verschiedene innovative Konzepte, wie z. B. das „home treatment" Praxistauglichkeit erlangen [8, 9]. In dem Zusammenhang verdient die S3Leitlinie „Psychosoziale Therapien bei schweren psychischen Erkrankungen“, die demnächst neu aufgelegt wird, besondere Erwähnung [5]. In dieser Leitlinie wird die Evidenzbasierung eines breiten Spektrums entsprechender Maßnahmen ausführlich dargestellt (reichend von psychoedukativen Interventionen für Betroffene und Angehörige über Trainingsmaßnahmen von Alltagsund sozialen Fertigkeiten, Ergo-, Sport- und Bewegungstherapie bis hin zu Interventionen zur Stärkung des Selbsthilfepotenzials). Die meisten dieser Angebote sind bisher auf jüngere Klienten ausgerichtet, sodass eine Aufgabe für die kommenden Jahre darin besteht, hier den $\mathrm{Zu}$ gang für alt gewordene Betroffene zu erleichtern.

Ziel all dieser Bemühungen muss es sein, bei einer Gruppe von meist schwer beeinträchtigten, häufig sozial stigmatisierten Menschen die Symptomlast ihrer Leiden soweit möglich zu reduzieren und ihre Lebensqualität und gesellschaftliche Teilhabe zu fördern [13]. Wir möchten mit diesem Themenschwerpunkt dazu einen Beitrag leisten und den Leserkreis der
Zeitschrift für Gerontologie und Geriatrie über aktuelle Entwicklungen auf diesem Gebiet informieren. Über Rückmeldungen $\mathrm{zu}$ diesem Heft würden wir uns sehr freuen. Unser Dank gilt allen, die an diesem Schwerpunkt mitgewirkt haben, den Autoren und Reviewern und dem Lektorat. Wir wünschen den Leserinnen und Lesern der Zeitschrift eine gewinnbringende Lektüre der nachfolgenden Beiträge.
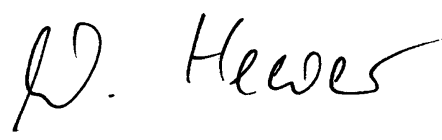

Walter Hewer

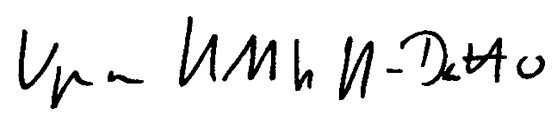

Vjera Holthoff-Detto

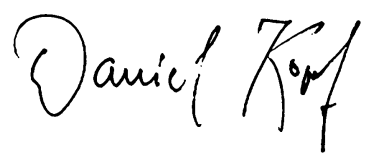

Daniel Kopf

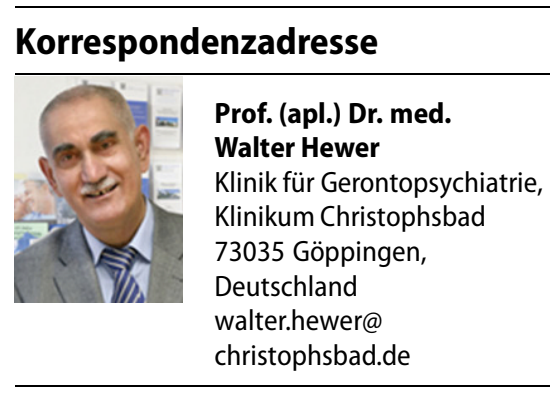

Interessenkonflikt. W. Hewer, V. Holthoff-Detto und D. Kopf geben an, dass kein Interessenkonflikt besteht.

\section{Literatur}

1. Amering M, Schmolke M (2012) Recovery. Das Ende der Unheilbarkeit. 5. Auflage. Psychiatrie-Verlag, Bonn

2. Andreas S, Schulz H, Volkert J et al (2017) Prevalence of mental disorders in elderly people: the European MentDis_ICF65+ study. Br J Psychiatry 210:125-131

3. Bundesministerium für Familie, Senioren, Frauen und Jugend (2015) Siebter Bericht zur Lage der älteren Generation in der BundesrepublikDeutschland. Bericht der Sachverständigenkommission an das Bundesministerium für Familie, Senioren, Frauen und Jugend. Der Deutsche Bundestag, Berlin

4. De Hert M, Correll CU, Bobes J et al (2011) Physical illness in patients with severe mental disorders. I.
Prevalence, impact of medications and disparities in health care. World Psychiatry 10:52-77

5. Falkai P (Hrsg) (2013) S3-Leitlinie Psychosoziale Therapien bei schweren psychischen Erkrankungen.Springer, Heidelberg, Berlin

6. Drake RE, Whitley R (2014) Recovery and severe mental illness: description and analysis. Can J Psychiatry 59:236-242

7. Gühne U, Becker T, Salize H-J, Riedel-Heller SG (2015) Wie viele Menschen in Deutschland sind schwer psychisch krank? Psychiatr Prax 42:415-423

8. Holthoff V (2015) Innovative Versorgungsstrategien in der Gerontopsychiatrie und -psychotherapie. Nervenarzt 86:468-474

9. Lang FU, GühneU, Riedel-Heller SG, BeckerT(2015) Innovative patientenzentrierte Versorgungssysteme. Nervenarzt 86:1313-1319

10. Meesters PD, Comijs HC, de Haan L et al (2011) Symptomatic remission and associated factors in a catchment area based population of older patients with schizophrenia. Schizophr Res 126:237-244

11. Meesters PD, de Haan L, Comijs HC et al (2012) Schizophrenia spectrum disorders in later life: prevalence and distribution of age at onset and sex in a Dutch catchment area. Am J Geriatr Psychiatry 20:18-28

12. Shefer G, Henderson C, Howard LM, Murray J, Thornicroft G (2014) Diagnostic overshadowing and other challenges involved in the diagnostic process of patients with mental illness who present in emergency departments with physical symptoms-a qualitative study. PLoS ONE 9(11):e111682. https://doi.org/10.1371/journal. pone. 0111682

13. Stengler (2018) Der DGPPN-Teilhabekompass II: Umsetzung sozialer Teilhabe für Menschen mit psychischen Erkrankungen. Nervenarzt. https:// doi.org/10.1007/s00115-018-0588-z

14. Volkert J, Schulz H, Härter M et al (2013) The prevalence of mental disorders in older people in Western countries-a meta-analysis. Ageing Res Rev 12:339-353 